Endocrinology

\section{Obesity and the insulin resistance syndrome}

\section{B Dunger}

\section{Commentary on the paper by Viner et al (see page 10)}

$\mathrm{T}$ he increasing prevalence of obesity during childhood in the UK has been highlighted by several recent publications $^{12}$ and has become the subject of considerable debate, not only in the media but also through the establishment of a select committee at the House of Commons and a working group of our own Royal College. The alarm bells have been ringing on the other side of the Atlantic for several years where the prevalence of obesity and type 2 diabetes during childhood has been increasing dramatically. ${ }^{3}$ A paper from the Yale group published in 2002 reported the presence of impaired glucose tolerance in $20-25 \%$ of obese children and adolescents and a further $4 \%$ had undiagnosed type 2 diabetes. ${ }^{4}$ Other reports from the Bogalusa cohort in the USA suggested that as many as $50 \%$ of overweight youngsters will have features of syndrome $X$ (the insulin resistance syndrome ${ }^{5}$ ). Thus, the report from Viner et al is both timely and relevant as it assesses the prevalence of these risk factors in obese UK children and adolescents. ${ }^{6}$

The authors report both prospective and retrospective data on 103 obese children and adolescents aged 2-18 years. They report that one third of these children had features of the insulin resistance syndrome. The occurrence of impaired glucose tolerance in this cohort despite, in many cases extreme obesity, was only around 11\% and none of the subjects had previously undiagnosed type 2 diabetes. This is perhaps encouraging given the high rates of impaired glucose tolerance reported in obese children from the USA and Far East. Nevertheless, overall the data should give us cause for concern as population studies indicate that these adverse features will track into adult life and, although it remains relatively rare, the first reports of type 2 diabetes occurring during childhood in the UK are now emerging.

Viner et al report data from a very selected population attending an obesity clinic at Great Ormond Street Hospital ${ }^{6}$ and, although their findings are similar to those reported by others who have set up similar clinics over the last five years, the data are not population based. We urgently need more epidemiological data from well constructed cohorts such as the Avon Longitudinal Study of Parents and Children. We also need to address the question as to what normative data are appropriate during childhood and adolescence. Normative data used in the present study are derived from multiple sources and do not necessarily take into account variation in insulin and lipid assays. There are no direct measures of insulin sensitivity, and the extent to which high insulin levels in obese children may reflect earlier maturation, increased statural growth, and increased lean body mass has not really been determined. In the present study, the authors were not able to detect any differences between ethnic groups, or between males and females, although such differences have been reported in many other studies during childhood and adolescence. Thus we cannot be certain that these data are representative of the general overweight obese population in the UK.

The data reported by Viner et al also highlight another intriguing observation. Some children, despite extreme obesity, do not appear to develop any features of the insulin resistance syndrome, nor do they appear to be at immediate risk of the development of type 2 diabetes. This may reflect the cross-sectional nature of the study and perhaps, as has been shown in studies from the USA, the greatest risk may be at puberty when gender and ethnic differences may become more evident. Certainly other data from paediatric and adult populations in the UK indicate that the risk of insulin resistance syndrome and type 2 diabetes may be greatest in children of South Asian descent. ${ }^{78}$ Cardiovascular disease risk is also greater in US blacks compared to white children. Such variation in disease risk may have its origins in pre-natal and early post-natal rates of weight gain or so called "thrifty genes". ${ }^{\text {? }}$ We must encourage debate on how we might change exercise patterns and improve the diets of children in order to reduce the overall burden of obesity. In addition, we need to identify factors which predispose to visceral fat deposition, insulin resistance syndrome, and impaired glucose tolerance, as such high risk subjects may need more targeted intervention.

Arch Dis Child 2005;90:1.

doi: 10.1136/adc.2003.046854

Correspondence to: Prof. D B Dunger, Addenbrooke's Hospital, Cambridge CB2 2QQ, UK; dbd25@cam.ac.uk

\section{REFERENCES}

1 Lobstein TJ, James WP, Cole TJ. Increasing levels of excess weight among children in England. Int J Obes Relat Metab Disord 2003;27:1136-8.

2 Reilly JJ, Dorosty AR, Emmett PM. Prevalence of overweight and obesity in British children: cohort study. BMJ 1999;319:1039.

3 Fagot-Campagna A, Pettitt DJ, Engelgau MM et al. Type 2 diabetes among North American children and adolescents: an epidemiologic review and a public health perspective. J Pediatr 2000;136:664-72.

4 Sinha R, Fisch G, Teague B, et al. Prevalence of impaired glucose tolerance among children and adolescents with marked obesity. N Engl J Med 2002;346:802-10.

5 Freedman DS, Dietz WH, Srinivasan SR, et al. The relation of overweight to cardiovascular risk factors among children and adolescents: the Bogalusa Heart Study. Pediatrics 1999;103(6 pt 1):1175-82

6 Viner RM, Segal TY, Lichtarowicz-Krynska E, et al. Prevalence of the insulin resistance syndrome in obesity. Arch Dis Child 2005;90:10-14.

7 Whincup PH, Gilg JA, Papacosta O, et al. Early evidence of ethnic differences in cardiovascular risk: cross-sectional comparison of British South Asian and white children. BM 2002;324:635-8.

8 Ramaiya KL, Kodali VRR, Alberti KGMM Epidemiology of diabetes in Asians of the Indian Subcontinent. Diabetes Metab Rev 1990;6: 125-46.

9 Ong KKL, Dunger DB. Thrifty genotypes and phenotypes in the pathogenesis of type 2 diabetes mellitus. J Paediatr Endocrinol Metab 2000;13:1419-24. 
Neurology

\section{Cerebral palsy: does SES make a difference?}

\section{P O D Pharoah}

\section{Commentary on the paper by Sundrum et al (see page 15)}

S ocial inequalities have a pervasive influence in almost all aspects of health and health service use. In many instances, as in smoking and lung cancer, the inequalities are a confounding variable and strategies of preventive action can address the primary cause. In those conditions where aetiology is uncertain, investigation of social inequalities may point to causal hypotheses and lead to successful preventive action.

Until 2001, the measures of social status used were the Registrar General's Social Class (RGSC) based on levels of occupational skill or the more detailed socioeonomic status (SES) classification that brought together occupations of similar social and economic status. ${ }^{1}$ Alternatively, proxy measures for affluence and deprivation based on census enumeration districts were applied to examine the association between incidence and prevalence rates of a large number of diseases. In the majority of instances, the association is an inverse one; the higher the social position or degree of affluence, the lower is the risk of disease.

In population studies of cerebral palsy there has been no consistent association observed. In this issue of Archives, Sundrum and colleagues examine the relation between cerebral palsy and socioeconomic status. ${ }^{2}$ The authors draw attention to this in the summary of these studies shown in table 1 of their paper. However, in this report of a population based study of cerebral palsy in West Sussex, they position themselves clearly on that side of the fence favouring a strong inverse association between RGSC and cerebral palsy prevalence. The title of the report is a misnomer because only RGSC and enumeration district deprivation quintiles and not SES are examined.

There are two main areas of this report where the observations made and conclusions drawn need to be tempered with caution. Primarily, the validity of the observation that there is a highly significant linear trend in association of RGSC and cerebral palsy needs to be examined. Secondly, if validity is established, the claim that $51 \%$ of cases of cerebral palsy were "attributable" to inequality in RGSC must be clarified.

The basis for the conclusion that the odds ratio for cerebral palsy prevalence has a linear association with RGSC arises from the data in table 2 of their paper. Four striking features are evident in this table:

- The very low prevalence of cerebral palsy in RGSC 1

- The high prevalence of cerebral palsy in RGSC Unclassified

- The high prevalence of cerebral palsy in those whose RGSC is missing

- The lack of a discernable trend in odds ratio between RGSC 2 through to RGSC 5.

The RGSC 1 and RGSC Unclassified are at opposite ends of the RGSC classification and, in doing the $\chi^{2}$ test for linear trend, the assumption is made that those whose RGSC is unclassified are at the lowest end of the social scalethat is, they are below RGSC 5. This is not a valid assumption and there are many reasons why the occupation may not be provided at the time of registration. It is pertinent that, when the RGSC unclassified group is omitted from the trend analysis, the trend becomes only marginally statistically significant with $\mathrm{p}=0.04$

The prevalence of cerebral palsy in RGSC missing is 10.3 per 1000 . This is a 4-5-fold increase when compared with general population cerebral palsy prevalence. The potential bias this group may induce becomes even more worrying in the light of the observation that RGSC was missing in $18.8 \%$ of cases of cerebral palsy but only in $4.1 \%$ of children without cerebral palsy. This difference is hugely significant $(p<0.0001)$. The RGSC was missing because the families, though resident in West Sussex, were born out of the county and the birth registration details were not available. Furthermore, this group had a disproportionate number of high risk infants (low birth weight, preterm, and multiple births). It is feasible that, if there is concern over the progress of a pregnancy, RGSC 1 mothers may be more likely than others to seek care or be referred outside the county, say to a teaching hospital. If so, there will be a deficit in cerebral palsy cases in RGSC 1 and the bias engendered would negate any social class trend observed.

In table 2, there is no discernible trend in odds ratios between RGSC 2 and RGSC 5. A $\chi^{2}$ for linear trend limited to these RGSC categories is non-significant $(p=0.94)$. It is clear that including RGSC 1 and RGSC Unclassified at opposite ends of the social spectrum is sufficient to convert a statistically non-significant trend into a significant one. However, the assumption that RGSC Unclassified is at the lowest end of the social spectrum is not valid and the potential for bias, particularly pertaining to RGSC 1, arising from the failure to record the occupation in a high proportion of children with cerebral palsy, means that any social class trend in the data needs greater justification.

As there is no trend within RGSC 2-5, it would be appropriate to test the null hypothesis that there is no difference in cerebral palsy prevalence of 1.1 per 1000 in RGSC 1 and 2.3 per 1000 in RGSC 2-5 combined. The null hypothesis must be rejected because the difference is highly statistically significant $(p=0.002)$. Is this an acceptable conclusion that RGSC 1 is somehow protected from cerebral palsy? If so, it is a unique observation and needs justification.

Cerebral palsy is not a single nosological entity. It is recognised that there is an inverse relation between birth weight and cerebral palsy prevalence due, in part, to the risk of very preterm infants sustaining perinatal hypoxic-ischaemic cerebral impairment. Also, the risk of preterm delivery is increased in the lower social classes. Therefore, in this preterm group, it is consistent that a social class gradient in cerebral palsy is observed. Indeed, after adjustment was made for birth weight and gestational age, the social class gradient in odds ratios was no longer significant.

Another group of cerebral palsies where a social class gradient may be expected are those acquired postneonatally, usually as a result of accidental and non-accidental head injury and severe neurological and other infections. These comprise $3-13 \%$ of all cases. ${ }^{3}$ The authors were unable to differentiate these postneonatally acquired cases in their database but, if they could be excluded, any social class trend in the remaining data would be limited further. 
Finally, the claim is made that $51 \%$ of cases of cerebral palsy were statistically "attributable" to inequalities in RGSC. This calculation is based on the assumption that a cerebral palsy prevalence of 1.1 per 1000 in RGSC 1 is a valid estimate and doubts over this estimate already have been referred to. The calculation also assumes that the prevalence of cerebral palsy in all other RGSCs could be reduced to 1.1 per 1000 . In addition to the validity of these assumptions being suspect, although the authors make it clear that the attribution is statistical, the reader frequently considers that the association demonstrated infers causation. This is not so; any observed epidemiological association cannot be assumed to be causal. Hill enumerated several criteria that justified an epidemiological association being considered causal, one of which is that the association be consistently observed in a variety of studies in different locations. ${ }^{4}$ Such consistency is lacking in studies reporting the association of cerebral palsy with social inequalities.

Whether or not socioeconomic status has a role in cerebral palsy is contentious and the authors' attempt to shed light on the problem is admirable, particularly since they were endeavouring to use routine data sources to address the issue. Efforts to replicate the study in other districts based on similar data sources are highly recommended.
Arch Dis Child 2005;90:2-3.

doi: 10.1136/adc.2004.052902

Correspondence to: Prof. P O D Pharoah, Department of Public Health, FSID Unit of Perinatal \& Paediatric Epidemiology, Muspratt Laboratory, Liverpool L69 3GB, UK; p.o.d.pharoah@liv.ac.uk

\section{REFERENCES}

1 Rose D, Prevalin DJ, eds. A researcher's guide to the National Statistics Socio-economic Classification. London: Sage Publications, 2003.

2 Sundrum R, Logan S, Wallace A, et al. Cerebral palsy and socioeconomic status: a retrospective cohort study. Arch Dis Child 2005;90:15-18.

3 Paneth N, Kiely JL. The frequency of cerebral palsy: a review of population studies in industrial nations since 1950. Clin Dev Med 1984:87:46-56.

4 Hill $\mathbf{A B}$. The environment and disease: association or causation. Proc $R$ Soc Med 1965;58:295-300.

\section{Status epilepticus with fever: how common is meningitis?}

\section{R Kneen, R Appleton}

\section{Commentary on the paper by Chin et al (see page 66)}

$\mathrm{T}$ he majority of febrile seizures are simple (generalised and brief, lasting less than 10 minutes), occurring in children aged 6 months to 5 years. The risk of acute bacterial meningitis $(\mathrm{ABM})$ in association with simple febrile convulsions is reported to be about $1-2 \% .^{1}$ Approximately one quarter of febrile convulsions are complex, defined as prolonged ( $>10$ minutes), having a focal onset, recurring in the same illness, or followed by a neurological deficit. It is generally believed that the risk of $\mathrm{ABM}$ with a complicated febrile seizure is higher than with a simple febrile convulsion, but the exact figure is not known. At the most severe end of the spectrum are children with convulsive status epilepticus (CSE) and fever, usually defined on the basis of a seizure lasting more than 30 minutes or a series of seizures lasting at least 30 minutes from which they do not regain consciousness in between. There are many causes of CSE with fever, but it is important to consider and identify those for which delayed treatment may have deleterious consequencesspecifically all central nervous system infections, including ABM, cerebral abscess, viral meningoencephalitis, tuberculous meningitis, and other less common infections.
Chin et al have studied children with CSE with fever as part of an ongoing study into status epilepticus, the North London convulsive STatus EPilepticus in childhood Surveillance Study (NLSTEPSS). ${ }^{2}$ Until recently, little information has been available on how likely this group of children are to have ABM. The authors have identified that $15-18 \%$ of children presenting with CSE and fever have ABM, but this is may be an underestimate as only one third of patients in the study underwent cerebrospinal fluid (CSF) analysis. The authors have also shown that children with ABM may not show the typical clinical signs of meningeal irritation, a well recognised observation in young children. The authors conclude that in their population, a significant number of children with CSE and fever were not investigated and treated for ABM. Although encephalitis (herpes simplex encephalitis) was not included in the study, it would seem appropriate to adopt a similar approach to the investigation (and management) of possible encephalitis as well as ABM.

The initial management of any child with a prolonged seizure and specifically CSE, involves resuscitative measures with particular emphasis on ensuring a secure airway, breathing, and circulation, and measurement of blood glucose and electrolytes and acid-base status. Subsequent management should focus on attempting to terminate the seizure and identify and treat any underlying cause. There is a UK based protocol for the management of a presenting tonicclonic seizure, including CSE, ${ }^{3}$ but it is unclear as to how closely this protocol is being followed in terms of both the drugs (and their doses) and the timing of their administration. This important information may become available from future analysis of data arising from NLSTEPSS. This is important as adult evidence suggests that the longer the tonic-clonic seizure (and episode of CSE), the more difficult it may be to terminate ${ }^{4}$ - and there is no reason to suspect that this would be any different in children. Consequently, anticonvulsant medication should be instituted in any child who has been seizing for more than five minutes and subsequent management should adhere to the published protocols. ${ }^{35}$ As yet, there is no recommended or consensus protocol for the treatment of refractory CSE, the definition of which is unclear, but usually includes persistence of the status following initial treatment with a benzodiazepine, phenytoin/phenobarbitone, or both. Current treatment options for treating refractory CSE include continuous intravenous infusions of either midazolam or diazepam, or anaesthesia with thiopentone or propofol with, ideally, electroencephalographic (EEG) monitoring (a full discussion of these treatment options is not within the remit of this commentary). The child with refractory CSE should always be discussed with a paediatric neurologist and should be transferred to a paediatric intensive care unit for both management of the persistent status and, where 
necessary, ongoing identification of the underlying cause. There is a low but definite mortality and morbidity in children with refractory CSE, and this reflects not only the underlying aetiology but also its management.

Investigation and treatment of an underlying cause of the febrile CSE, and specifically an underlying CNS infection, must always be considered. Chin et al found that less than a third of patients in their study had a lumbar puncture (LP) and only two thirds were treated with antibiotics at presentation. ${ }^{2}$ There does appear to be an increasing reluctance of paediatricians to undertake an LP in children with suspected CNS infection, despite the fact that CSF analysis findings are often helpful and may influence patient management. ${ }^{6} \mathrm{~A}$ prolonged seizure is thought to increase intracranial pressure, ${ }^{7}$ making it a contraindication to LP. In addition, ABM itself is known to cause coning, ${ }^{8}$ and LP may precipitate cerebral herniation in some severe cases of ABM. ${ }^{9}$ For these reasons it would be unwise to undertake an LP immediately after cessation of the CSE. It is our practice to treat these children with appropriate parenteral antibiotics, as well as intravenous acyclovir if herpes encephalitis is considered a likely diagnosis, until it is considered safe to undertake an LP.

The difficulty is deciding precisely when an LP should be undertaken; this is likely to be different for each patient. Arguably, it should be performed as soon as it is clear there is no medical contraindication. In practice, this may vary from 30 to 60 minutes following cessation of the episode of CSE to one or two days after presentation. In some cases it will rapidly become apparent that the child has no signs of CNS infection (for example, those with a complex febrile convulsion who make a rapid recovery). However, if the child remains continuously or intermittently febrile or unwell, and recovery is less rapid or there is any doubt about ABM or encephalitis, CSF analysis is likely to provide useful information. ${ }^{6}$ If the child requires admission to intensive care for persisting seizure activity, or the child shows any abnormal neurological features (cerebral irritability, neurological asymmetry, or abnormal behaviour), neuroimaging should be undertaken prior to LP because patients may be too sedated to accurately assess the level of coma. Although it is recognised that normal brain computed tomography (CT) cannot exclude raised intracranial pressure, it will exclude focal pathology, including a cerebral abscess or a subdural collection and, using contrast, may also show meningeal enhancement. ${ }^{10}$

The treatment of an underlying cause, and specifically presumed ABM or herpes encephalitis, must again be initiated early using appropriate antibiotics and acyclovir in appropriate doses. The initial choice of antibiotic may need to be revised, depending on the results of bacterial/viral antigen studies and also blood, other tissue, and CSF cultures. Without information from CSF analysis (cell count, protein and glucose levels (and simultaneous blood glucose); culture and sensitivities), treatment of a presumed ABM or herpes encephalitis is likely to be empiric or even blind-with consequent uncertainty on the duration of treatment with intravenous antibiotics or acyclovir, or both.

Children with proven ABM should, simultaneously with antibiotics, receive corticosteroids. ${ }^{11}$ Despite the lack of evidence, it is our practice to also use corticosteroids (either dexamethasone or prednisolone) in the treatment of proven or probable herpes encephalitis. The duration of steroid treatment varies; our practice is to treat for a minimum of seven days in proven ABM or herpes encephalitis

Febrile CSE is a relatively common paediatric emergency. Acute symptomatic (specifically due to an acute CNS infection) and prolonged febrile seizures are the most causes of febrile CSE. Its management must be timely and appropriate to reduce the risk of death and irreversible neurological sequelae. The study by Chin et al has highlighted differences in the management of febrile CSE due to ABM between individual units and individual children. ${ }^{2}$ The risk of herpes simplex encephalitis in this group of patients and its management are unknown, and the empirical treatment with acyclovir in this clinical situation is controversial. It might be possible for the authors to investigate the risk of viral encephalitis in the ongoing NLSTEPS Study. In this commentary we have attempted to address some of the more important issues with additional information about our own practice. Clearly, there is an indication for trying to develop evidence based guidelines to optimise the investigation and treatment of refractory febrile CSE.

Arch Dis Child 2005;90:3-4.

doi: 10.1136/adc.2004.055442

\section{Authors' affiliations}

R Kneen, R Appleton, The Roald Dahl EEG

Unit, Department of Neurology, Royal Liverpool Children's Hospital (Alder Hey), Liverpool, UK

Correspondence to: Dr R Appleton, Royal Liverpool Children's Hospital, Alder Hey, Liverpool L12 2AP, UK; richard.appleton@ rlch-tr.nwest.nhs.uk

\section{REFERENCES}

1 Carroll W, Brookfield D. Lumbar puncture following febrile convulsion. Arch Dis Child 2002;87:238-40.

2 Chin RFM, Neville BGR, Scott RC. Meningitis is a common cause of convulsive status epilepticus with fever. Arch Dis Child 2005;90:66-9

3 Appleton R, Choonara I, Martland T, et al. The treatment of convulsive status epilepticus in children. The Status Epilepticus Working Group. Arch Dis Child 2000;83:415-19.

4 Treiman DM. Generalised convulsive status epilepticus in the adult. Epilepsia 1993;34(suppl 1):2-11.

5 Advanced Life Support Group. Advanced paediatric life support, 3rd edn. London: BMJ Publishing Group, 2001:139-47.

6 Kneen R, Solomon T, Appleton R. The role of lumbar puncture in suspected CNS infection-a disappearing skill? Arch Dis Child 2002;87:181-3.

7 Minns RA, Brown JK. Intracranial pressure changes associated with childhood seizures. Dev Med Child Neurol 1978:20:561-9.

8 Horwitz SJ, Boxerbaum B, O'Bell J. Cerebral herniation in bacterial meningitis in childhood. Ann Neurol 1980;7:524-8.

9 Slack J. Deaths from meningococcal infection in England and Wales in 1978. J R Coll Physicians Lond 1982;16:40-4.

10 Mellor D. The place of computed tomography and lumbar puncture in suspected meningitis. Arch Dis Child 1992:67:1417-19.

11 de Beek D, de Gans J, Mclntyre P, et al. Corticosteroids in acute bacterial meningitis (Cochrane Review). In: The Cochrane Library Issue 2. Chichester, UK: John Wiley and Sons, 2004. 